\title{
Erratum: Collective dynamics of soft active particles [Phys. Rev. E 91, 032706 (2015)]
}

\author{
Ruben van Drongelen, Anshuman Pal, Carl P. Goodrich, and Timon Idema \\ (Received 5 December 2016; published 27 December 2016)
}

DOI: 10.1103/PhysRevE.94.069904

There is a missing normalization factor in the last term of Eq. (4) of the paper. Particles align with the preferred orientation of their neighbors, not with all neighbors individually. This effectively means that this term needs to be normalized by the number of neighbors. The text referring to the equation, as well as the values of the parameters, are correct.

The equation should read

$$
\begin{aligned}
T_{i} & =T_{i, \text { boundary }}+T_{i, \text { noise }}+T_{i, \text { align }} \\
& =T_{\text {in }} \Delta \theta_{i} \cdot \Theta\left(\theta_{\text {out }, i}-\pi\right)+T_{\text {noise }} \xi_{i}+\frac{T_{\text {align }}}{\left|\mathcal{N}_{i}\right|} \sum_{j \in \mathcal{N}_{i}} \Delta \psi_{i j},
\end{aligned}
$$

where the added factor $\left|\mathcal{N}_{i}\right|$ denotes the size of the set, or equivalently the number of neighbors of particle $i$.

We want to thank Marlon Röben and Jan-Frederik Pietschmann for pointing out this mistake. 